\title{
Sustainable Production of Furfural in Biphasic Reactors Using Terpenoids and Hydrophobic Eutectic Solvents
}

\author{
Andrés Cañada-Barcala, Diego Rodríguez-Llorente, Laura López, Pablo Navarro, Elisa Hernández, \\ V. Ismael Águeda, Silvia Álvarez-Torrellas, Juan C. Parajó, Sandra Rivas, and Marcos Larriba*
}

Cite This: ACS Sustainable Chem. Eng. 2021, 9, 10266-10275

Read Online

\section{ACCESS | Llll Metrics \& More | 回 Article Recommendations | (s) Supporting Information}

ABSTRACT: Furfural comes from lignocellulosic biomass that, together with its derived products, has many useful applications in several industries. Furfural is usually obtained via pentose dehydration using a biphasic reactor to extract furfural in situ from its reaction medium. However, the conventional solvents used so far, mainly toluene and methyl isobutyl ketone (MIBK), are harmful to health and the environment. Therefore, hydrophobic natural solvents have been successfully tested in this work to improve the performance of the process from a sustainable point of view. First, 30 natural solvents were screened using the conductor-like screening model for real solvents (COSMO-RS) method to select solvents with high affinity to furfural. From the

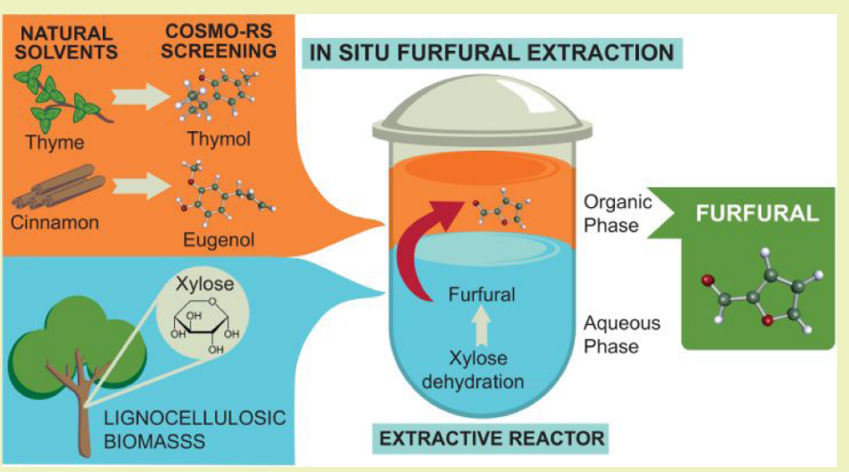
results obtained in the screening, 14 natural and 2 conventional solvents were selected for experimentation, including thymol, eugenol, toluene, MIBK, and several hydrophobic eutectic solvents formed by thymol. Then, the liquid-liquid extraction of furfural was carried out both in vials and in a reactor, simulating the usual temperature and $\mathrm{pH}$ conditions for obtaining furfural. Thymol and eugenol showed extraction yields of 95 and $91 \%$, significantly higher than those of conventional solvents MIBK and toluene, which were 85 and $81 \%$, respectively. Finally, the in situ reaction and extraction of furfural from xylose were performed using the natural solvents eugenol and thymol and the conventional solvent MIBK. Under operating conditions, namely, microwave heating to $443.2 \mathrm{~K}, 10 \mathrm{~min}$ of reaction, and a solvent-to-feed ratio of 1.00 , xylose conversion of $96.7 \%$, furfural selectivity of $75.3 \%$, and a furfural production yield of $72.8 \%$ from xylose were obtained using eugenol as the organic solvent, with improved outcomes over MIBK and thymol cases, pointing an adequate approach to improve both the effectiveness and the sustainability of the process.

KEYWORDS: furfural, COSMO-RS, biphasic reactor, terpenoids, in situ reaction

\section{INTRODUCTION}

Furfural (2-furaldehyde) is a versatile organic compound, employed in the synthesis of bio-based chemicals, fuel precursors, and solvents. ${ }^{1}$ Some furfural-derived compounds are furfuryl alcohol, methylfuran, tetrahydrofuran, methyltetrahydrofuran, tetrahydrofuryl alcohol, and furoic acid. ${ }^{2-5}$ Furfural and its derivatives have several industrial applications, ${ }^{1,6}$ including fungicides and nematicides, transportation fuels, gasoline additives, lubricants, resins, decolorizing agents, jet-fuel-blend stocks, drugs, insecticides, bioplastics, and flavor enhancers for food and drinks. ${ }^{7}$

Furfural is produced from vegetal biomass, a sustainable resource that is gaining importance as an alternative industrial feedstock to limit the dependence on non-renewable feedstock and energy inputs. ${ }^{7,8}$ In this context, the US Department of Energy has considered furfural as one of the topmost 30 chemicals that can be produced from biomass. ${ }^{9,10}$ Interestingly, furfural can be produced in lignocellulose biorefineries, which are focused on the sustainable processing of biomass into a spectrum of marketable products and bioenergy. ${ }^{11,12}$

Furfural is commercially produced by acid-catalyzed hydrolysis-dehydration of the pentosans present in vegetal biomass. Xylan is the most abundant pentosan, and its hydrolysis results in the formation of xylose, which can be dehydrated into furfural at high temperatures. ${ }^{6,11,13}$ No direct synthetic method has been found for the production of furfural. $^{14}$ The main drawback of the commercial furfural production processes is the participation of side reactions leading to the formation of black, resinous products called humins. These side reactions include furfural condensation and

Received: April 26, 2021

Revised: July 6, 2021

Published: July 19, 2021 
reactions of furfural with its intermediates (furfural resinification), ${ }^{15}$ which are responsible for decreased yields and poor selectivities.

The results reported for furfural production in aqueous media can be improved by using biphasic systems, where the furfural generated in the aqueous phase is continuously transferred to the organic one, limiting the extent of the aforementioned side reactions. ${ }^{16}$ Other advantages of biphasic operation are the thermodynamic shifting of the reaction toward the products and the purification-concentration effects resulting from the extraction. ${ }^{12}$ A number of studies dealing with furfural production in biphasic systems have been reported recently. ${ }^{15,17-21}$

The type of solvent employed in the biphasic operation is an important variable affecting the production and recovery of furfural. For this purpose, literature reports the utilization of ketones [e.g., methyl isobutyl ketone (MIBK) $],{ }^{17-20}$ alcohols (e.g., 1-butanol and 2-butanol), ${ }^{22}$ aromatics (e.g., toluene), ${ }^{21}$ lactones (e.g., $\gamma$-valerolactone), ${ }^{23}$ ethers (e.g., tetrahydrofuran and methyltetrahydrofuran), deep eutectic solvents (DES), ${ }^{24-26}$ and ionic liquids ${ }^{3,26,27}$ In this field, the integration of new tools such as the COSMO-RS (conductor-like screening model for real solvents) method to predict the behavior of solvents in biphasic media (including their ability to extract furans) was recently reviewed by Esteban et al. ${ }^{12}$

Regarding the liquid-liquid extraction of furfural from reaction media, terpenes, terpenoids, and natural eutectic solvents stand out as promising separation agents in terms of efficiency and sustainability, based on their environmentally friendly character, natural origin, high availability and versatility, and negligible toxicity to mammals. ${ }^{8,28,29}$

Terpenes and terpenoids are one of the widest groups of natural compounds. They are mainly obtained from plants and also from insects, marine fungi, and micro-organisms such as algae and protozoa. ${ }^{30}$ For example, thymol is obtained from thyme and eugenol from cinnamon. Terpenes are used in cosmetic, flavor, or fragrance industries, as well as in medical and pharmaceutical applications, due to their anti-inflammatory, anti-oxidant, and anti-microbial activities. ${ }^{31-33}$ The use of terpenes and terpenoids as solvents is increasing due to their biodegradability, versatility, and competitive cost in comparison with petroleum-derived solvents. ${ }^{34}$ The current applications of terpenes and terpenoids include the extraction of lipids from microalgae, ${ }^{34,35}$ manufacturing of sustainable polymers, ${ }^{36}$ and extraction of volatile fatty acids, ${ }^{37}$ phenols, ${ }^{38}$ and alcohols. $^{39}$ Menthol, carvacrol, and thymol, among others, have been tested by Dietz et l. $^{40}$ for furfural extraction from reaction media. In this study, the generation and in situ extraction of furfural from xylose in reaction media containing thymol were reported to proceed with comparatively low furfural degradation.

In the past few years, increasing attention has been devoted to the utilization of eutectic solvents obtained from natural compounds (natural deep eutectic solvents, NADES). Eutectic solvents are a mixture of two or more compounds that present a depression in the melting point resulting from the hydrogen bonding interactions between a hydrogen bond donor and an acceptor. $^{41}$ In particular, NADES present advantages in comparison with conventional solvents and ionic liquids, including increased biodegradability, lower toxicity, lower environmental impact, and easy preparation. ${ }^{42-46}$ Recently, hydrophobic eutectic solvents have been formulated with terpenes and terpenoids together with acids such as caprylic acid $\left(\mathrm{C}_{8} \mathrm{OOH}\right)$, capric acid $\left(\mathrm{C}_{10} \mathrm{OOH}\right)$, and lauric acid $\left(\mathrm{C}_{12} \mathrm{OOH}\right){ }^{28,37,47,48}$

This work deals with the evaluation of natural solvents as components of biphasic media for the in situ production and separation of furfural. First of all, simulation with the COSMORS method was carried out to identify the ones with a higher potential. ${ }^{49,50}$ The 16 most promising solvents identified from the COSMO-RS assessment were experimentally tested for furfural extraction. The best natural solvents, thymol and eugenol, were used in additional experiments performed in a reactor under the conditions used for furfural production, and their thermal stability was studied by FTIR (Fourier-transform infrared) spectroscopy. Similar experiments were performed with MIBK for comparative purposes. In order to confirm the technical feasibility of the proposed process, reaction media containing selected natural solvents were employed for the simultaneous production and extraction of furfural from xylose, operating in batch or microwave reactors.

\section{EXPERIMENTAL SECTION}

Selection of Natural Solvents by Molecular Simulation with COSMO-RS. Solvents Tested in the Screening. Several solvents were selected to assess their suitability as agents for furfural separation and screened using COSMOtherm software. The activity coefficients of furfural at infinite dilution and $323.15 \mathrm{~K}$ were calculated for 5 terpenes ( $\alpha$-pinene, limonene, $\beta$-pinene, camphene, and myrcene), 14 terpenoids (fenchol, citronellol, myrtenol, menthol, verbenol, eucalyptol, geraniol, isopulegol, linalool, citral, citronellal, thujone, eugenol, and thymol), 11 hydrophobic eutectic solvents [(menthol + camphor $),($ menthol + borneol $),\left(\mathrm{C}_{12} \mathrm{OOH}+\right.$ menthol $),\left(\mathrm{C}_{10} \mathrm{OOH}+\right.$ menthol), $\left(\mathrm{C}_{8} \mathrm{OOH}+\right.$ menthol $),($ thymol + camphor $),($ thymol + borneol), (thymol + menthol), $\left(\mathrm{C}_{12} \mathrm{OOH}+\right.$ thymol $),\left(\mathrm{C}_{10} \mathrm{OOH}+\right.$ thymol), and $\left(\mathrm{C}_{8} \mathrm{OOH}+\right.$ thymol $\left.)\right]$, and 3 conventional solvents (1butanol, toluene, and MIBK) that are considered as benchmarks.

Computational Details. The affinity of furfural for these solvents was predicted using COSMOtherm software (version C3.0, release 12.01). ${ }^{49}$ Lower values of the activity coefficients of furfural at infinite dilution $\left(\gamma^{\infty}\right)$ indicated higher furfural solubilities and so higher extraction yields. The COSMO continuum solvation model at the BVP 86/TZVP/DGA1 ${ }^{51}$ calculation theory level and BP_TZVP_C30_1201 ${ }^{52}$ in the parameters setting were used for the chemical calculations. This prediction was previously suitable for selecting the best terpenes and hydrophobic eutectic solvents for the liquid-liquid extraction of phenols and volatile fatty acids. ${ }^{37,38}$ The polarized charge density $(\sigma)$ profiles of the solvents selected for the liquid-liquid extraction of furfural were also obtained to assess the interactions between furfural and the conventional and natural solvents tested.

The molecular conformation of the solvents employed in this study was optimized using the BP86/TZVP computational level with Turbomole 4.2 software, to obtain a COSMO file suitable for the COSMOtherm analysis. As reported in previous studies, ${ }^{37,38}$ the calculations for the eutectic solvents were performed considering each one as two different compounds mixed at the eutectic composition.

The COSMO-RS method has also been used to predict the interactions between furfural and solvents. For this purpose, the excess enthalpies of equimolar mixtures of furfural with the two best terpenoids in furfural extraction (eugenol and thymol) and with the two conventional organic solvents (MIBK and toluene) have been estimated. The contributions of hydrogen bonding, van der Waals, and misfit forces on the excess enthalpies have been determined.

Chemicals. COSMOtherm screening enabled the selection of 16 solvents, which were employed for furfural extraction. Tables S1 and S2 of the Supporting Information list information on the structures, purities, and suppliers of the chemicals employed in experiments. The aqueous solutions were made with ultrapure water from Purelab flex Elga Veolia water purification system. 
Eight selected eutectic solvents were prepared at the eutectic point compositions as follows: 0.66 molar of caprylic acid in $\left(\mathrm{C}_{8} \mathrm{OOH}+\right.$ thymol), ${ }^{47} 0.70$ molar of menthol in (menthol + borneol), ${ }_{8}^{47,53} 0.44$ molar of lauric acid in $\left(\mathrm{C}_{12} \mathrm{OOH}+\right.$ thymol $)$, and equimolar proportions in (menthol + camphor), (thymol + menthol), (thymol + camphor), $\left(\mathrm{C}_{10} \mathrm{OOH}+\right.$ thymol $)$, and (thymol + borneol). ${ }^{47,48,53}$ The eutectic solvents were prepared at $323.2 \mathrm{~K}$ in a Selecta Tectron Bio thermostat under stirring until a colorless liquid was obtained.

Liquid-Liquid Extraction of Furfural from an Aqueous Solution. Synthetic aqueous solutions containing $10 \mathrm{~g}$ furfural/L were employed in experiments, since this is the usual concentration of furfural in reaction media. ${ }^{19}$ The natural solvents were assayed as agents for furfural extraction operating at a solvent-to-feed $(\mathrm{S} / \mathrm{F})$ ratio in a volume of $0.33,0.50,1.00$, or 2.00 . The mixture of the furfural solution and the solvent, with a total volume of $5 \mathrm{~mL}$, were placed in 8 $\mathrm{mL}$ vials and stirred at $800 \mathrm{rpm}$ in a dry bath IKA C-MAG HS for 12 $\mathrm{h}$ at $323.2 \pm 0.5 \mathrm{~K}$ and atmospheric pressure and then kept without stirring for $12 \mathrm{~h}$ at the same temperature and pressure to allow the separation of phases. The aqueous and organic phases were analyzed in a Varian CP-3800 gas chromatograph coupled with a flame ionization detector and an Agilent CP-WAX column $(30 \mathrm{~m} \times 0.530$ $\mathrm{mm}$ ). Analyses were performed using $\mathrm{He}$ as a carrier gas (flow, $2 \mathrm{~mL} /$ min) keeping the oven at $443.2 \mathrm{~K}$. The water content of the solvent phase was measured using a Mettler Toledo DL 32 Karl Fischer coulometer.

Liquid-Liquid Extraction of Furfural in a Batch Reactor under Reaction Conditions. The best natural solvents identified from the previous experiments, thymol and eugenol, were used for furfural extraction in experiments using a $100 \mathrm{~mL}$ Berghof reactor at controlled temperature and stirring. The assays were performed under operational conditions typically employed for furfural production $(443.2 \pm 0.5 \mathrm{~K}$, autogenous pressure) in the absence or presence of $0.1 \mathrm{M}$ sulfuric acid. ${ }^{19}$ In these experiments, $15 \mathrm{~mL}$ of aqueous furfural and $15 \mathrm{~mL}$ of solvent were kept in the reactor for $2 \mathrm{~h}$ under stirring (800 rpm).

The chemical and thermal stability of the solvents was assessed by attenuated total reflection with Fourier transform infrared (ATRFTIR) spectroscopy, proton nuclear magnetic resonance ( ${ }^{1} \mathrm{H}$ NMR), and thermogravimetric analysis (TGA). The spectra of thymol and eugenol were recorded using a Nicolet iS50 spectrometer with a SpectraTech ATR performer. The data were recorded for samples of thymol and eugenol withdrawn before and after heating, in the wavenumber range $500-4000 \mathrm{~cm}^{-1}$. To check the thermal stability of the solvents, in addition to FTIR analysis, ${ }^{1} \mathrm{H}$ NMR was used. For this purpose, $20 \mu \mathrm{L}$ of each extract was dissolved in $0.5 \mathrm{~mL}$ of deuterated acetone and analyzed by ${ }^{1} \mathrm{H}$ NMR using a Bruker Varian Unity 500 . In addition to studying the chemical stability of the solvents by FTIR and ${ }^{1} \mathrm{H}$ NMR, the thermal stability of the solvents has been analyzed by TGA. For this purpose, the TGA-LABSYS evo DTA/DSC equipment has been used for dynamic analysis with a heating rate of $10 \mathrm{~K} / \mathrm{min}$ from 303.2 to $1073.2 \mathrm{~K}$, under a He atmosphere with a flow rate of $30 \mathrm{~mL} / \mathrm{min}$.

In Situ Reaction and Extraction of Furfural from Biphasic Reaction Media Containing Xylose. Xylose, a hemicellulose hydrolysis product, was employed as a model substrate for furfural production. The reaction media were made of an aqueous xylose solution $(139.40 \mathrm{mmol} / \mathrm{L})$, sulfuric acid (the catalyst facilitating xylose dehydration into furfural, employed at a concentration of $0.1 \mathrm{M}$ with respect to the aqueous phase), and a natural solvent (eugenol or thymol). Similar experiments were performed with MIBK, considered as a reference solvent. The total volume of phases was $30 \mathrm{~mL}$, and the solvent-to-feed ratio was 1.00 in volume. The experiments were done in duplicate. The compositions were measured by HPLC (highperformance liquid chromatography) and GC (gas chromatography), as described below.

Operation in a Reactor with Conventional Heating. Samples of the acidic aqueous phase $(15 \mathrm{~mL})$ and the considered solvent $(15$ $\mathrm{mL}$ ) were mixed in a $0.1 \mathrm{~L}$ stainless-steel reactor from Parr and stirred at $400 \mathrm{rpm}$. The reactor was heated up to $443.2 \mathrm{~K}(t=0 \mathrm{~min})$, and this temperature was kept for the preset reaction times $(15,30,45,60$, or $90 \mathrm{~min}$ ). The time needed to reach the target temperature from $333.2 \mathrm{~K}$ was $9 \mathrm{~min}$ and $50 \mathrm{~s}$. At the end of the reaction, the reactor was cooled, and the organic and aqueous phases were separated in a separatory funnel. The aqueous phases were diluted and filtered through $0.45 \mu \mathrm{m}$ membranes and analyzed by HPLC using a 1200 series Agilent chromatograph fitted with a refractive index detector and a diode array detector. The column used for analysis was a $300 \times$ $7.8 \mathrm{~mm}$ Aminex HPX-87H, eluted with $3 \mathrm{mN} \mathrm{H}_{2} \mathrm{SO}_{4}$ at $0.6 \mathrm{~mL} / \mathrm{min}$ and $323 \mathrm{~K}$. Xylose and furfural were determined from data obtained with the refractive index detector and the diode array detector $(280$ $\mathrm{nm}$ ), respectively. The organic phases were also analyzed by GC as explained above.

Operation in a Reactor with Microwave Heating. Samples of the acidic aqueous phase $(15 \mathrm{~mL})$ and solvent $(15 \mathrm{~mL})$ were mixed in $0.1 \mathrm{~L}$ stirred Teflon vessels and heated in a MARS6 CEM Corporation microwave accelerated reaction system. The media were heated from 333.2 to $443.5 \mathrm{~K}$ in $3 \mathrm{~min}$ and $30 \mathrm{~s}$ (power, $430 \mathrm{~W}$ ). Once the reactor reached $443.2 \mathrm{~K}(t=0)$, the temperature was kept constant for the desired reaction times $(5,10,15,20,30$, or $45 \mathrm{~min})$. Then, the vessels were cooled, and the phases were separated in a separatory funnel. The aqueous and organic phases were analyzed by HPLC and GC, respectively, using the previously described methods. From the results obtained by HPLC and GC, xylose conversion $\left(X_{\mathrm{xyl}}\right)$, furfural selectivity $\left(S_{\text {furf }}\right)$, furfural production yield $\left(Y_{\text {furf }}\right)$, and furfural extraction yield $\left(\mathrm{Yld}_{\mathrm{ext}}\right)$ were calculated using the following equations

$$
\begin{aligned}
& X_{x y l}=\frac{C_{x, 0}-C_{x}}{C_{x, 0}} \times 100 \\
& S_{\text {furf }}=\frac{C_{F}^{\text {org }}+C_{F}^{\mathrm{aq}}}{C_{x, 0}-C_{x}} \times 100 \\
& Y_{\text {furf }}=\frac{C_{\mathrm{F}}^{\text {org }}+C_{\mathrm{F}}^{\mathrm{aq}}}{C_{x, 0}} \times 100 \\
& \text { Yld }_{\text {ext }}=\frac{C_{\mathrm{F}}^{\text {org }}}{C_{\mathrm{F}}^{\text {org }}+C_{\mathrm{F}}^{\mathrm{aq}}} \times 100
\end{aligned}
$$

where $C_{x, 0}$ is the initial xylose concentration in the aqueous phase, $C_{x}$ is the xylose concentration in the aqueous phase at the considered time, $C_{\mathrm{F}}^{\mathrm{org}}$ is the furfural concentration in the organic phase, and $C_{\mathrm{F}}^{\mathrm{aq}}$ is the furfural concentration in the aqueous phase.

Simulation of Furfural Separation from Eugenol and MIBK by Distillation. Conventional distillation was simulated in an Aspen Plus v11 commercial simulator to compare eugenol (NISTV110.NIST-TRC databank) and MIBK (APV110.PURE32 databank) solvents in the isolation of the product, furfural. A COSMO-SAC thermodynamic model developed by Lin and Sandler $(2002)^{54}$ was selected, and COSMOtherm v. 19.0.4 was used to calculate the desired properties of eugenol, namely, the sigma profile and the COSMO volume. After analyzing the boiling points of the solvents and furfural, both strategies are envisioned. Furfural is distilled from eugenol due to its higher volatility, whereas MIBK is distilled from furfural due to its lower boiling point. A Radfrac model was employed to simulate both distillations cases, described with 20 equilibrium stages and adjusting the reflux ratio to satisfy $99.9 \%$ mole purity of furfural, in the residue or distillate for the cases of MIBK and eugenol, respectively. The emulated mixture used as a benchmark, containing 0.02 and 0.98 molar fractions of furfural and solvent, respectively, was fed to the column at stage 10 at $443.2 \mathrm{~K}$ and 1 atm with a mole flow rate of $100 \mathrm{kmol} / \mathrm{s}$. These conditions are selected to ease the comparison between both solvents, creating a fair scenario for the separation evaluation. Atmospheric pressure was set to make the comparison easier in terms of energy consumption, avoiding the use of vacuum.

\section{RESULTS AND DISCUSSION}

Solvent Screening Using COSMO-RS. For the sake of selecting the most suitable natural solvent for furfural 


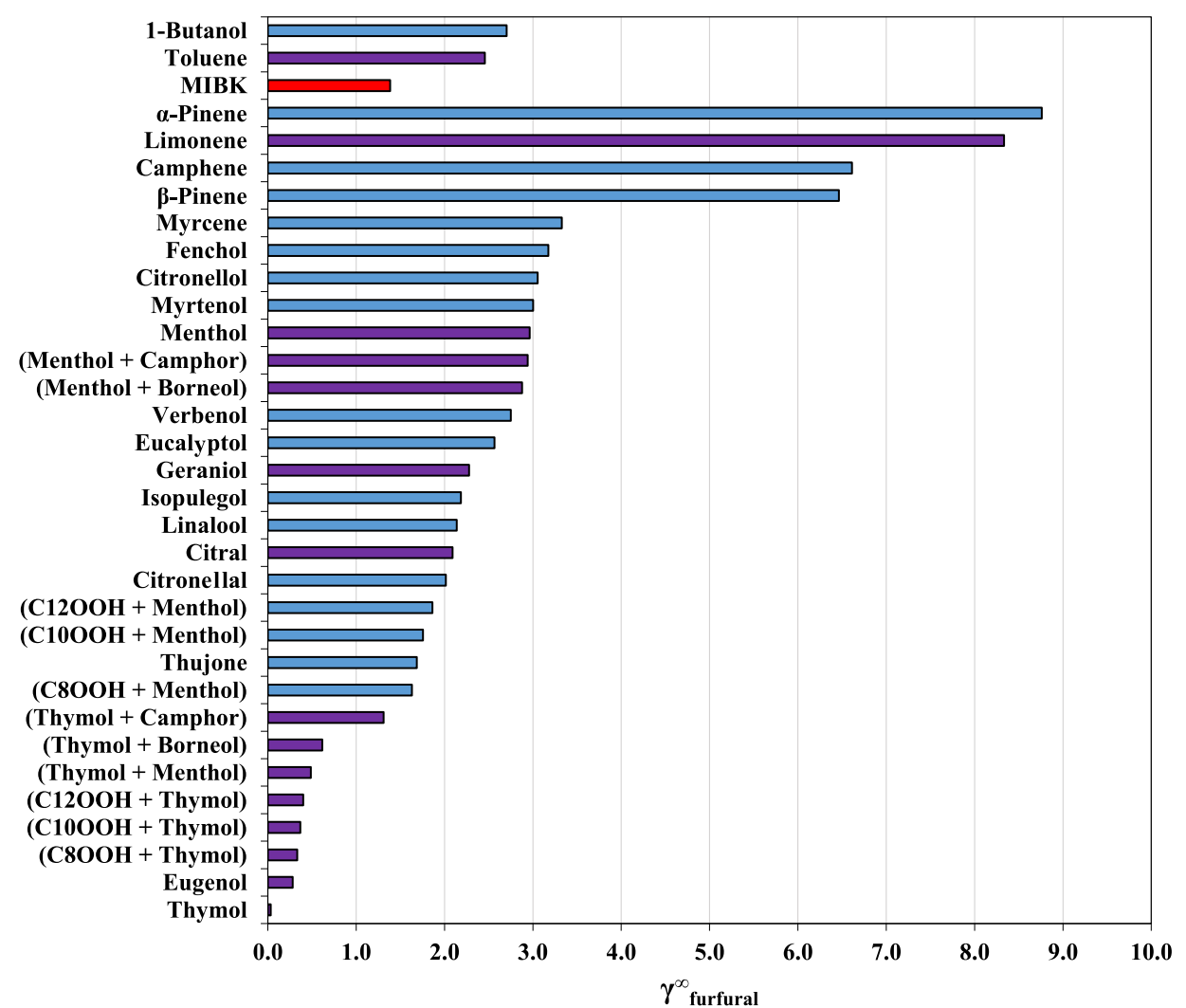

Figure 1. Activity coefficients at infinite dilution of furfural in terpenes, terpenoids, eutectic solvents, and conventional solvents (toluene, 1-butanol, and MIBK) calculated at $323.2 \mathrm{~K}$ using the COSMO-RS method.

extraction in its reaction medium, a screening of a wide range of natural solvents using COSMO-RS was performed. With this approach, the activity coefficients at infinite dilution of furfural in natural and conventional solvents were estimated at 323.2 K using COSMOtherm software. The numeric values of the activity coefficients of furfural at infinite dilution using terpenes, terpenoids, and eutectic and conventional solvents are depicted in Figure 1.

A low value of the activity coefficient at infinite dilution of furfural implies strong interactions with the solvent. Therefore, the solvents that have shown lower values of the activity coefficient at infinite dilution are expected to achieve high furfural extraction yields. The results obtained suggest that the terpenoids and eutectic solvents that are oxygenated alkenes and aromatics are the solvents with the best extraction performance to extract furfural. Thus, thymol, eugenol, and the eutectic solvents formed by thymol are the compounds that showed the lowest activity coefficient of furfural at infinite dilution. Also, the results suggest that eugenol, thymol, and the eutectic solvents with thymol would present a better performance than the conventional solvents MIBK, toluene, and 1-butanol.

To explain the observed trends, $\sigma$-profiles of furfural and the selected solvents were obtained using COSMOtherm software and are presented in Figures $\mathrm{S} 1-\mathrm{S} 3$ of the Supporting Information. The polarized charge density $(\sigma)$ values from -0.005 to $0.005 \mathrm{e} / \mathrm{A}^{2}$ correspond to the non-polar segments, while the charge density values under $-0.005 \mathrm{e} / \mathrm{A}^{2}$ and above $0.005 \mathrm{e} / \mathrm{A}^{2}$ correspond to the electrophilic (hydrogen bond donor) and nucleophilic (hydrogen bond acceptor) groups, respectively. Furfural shows a strong hydrogen bond donor segment due to the aldehyde group. Thus, the solvents that present hydrogen bond acceptor segments will present low furfural activity coefficients. Therefore, thymol and eugenol present strong acceptor hydrogen bond segments due to the alcohol group. Also, furfural and the solvents mentioned are aromatic compounds, presenting a cloud of $\pi$ electrons that increase the interactions and, therefore, would facilitate the solubility of furfural in the solvent with thymol and eugenol.

To predict solute-solvent interactions, the contributions of hydrogen bonding, van der Waals, and misfit forces on the excess enthalpies of an equimolar mixture of furfural and thymol or eugenol have been estimated by the COSMO-RS method. According to the results obtained in these calculations, in the case of eugenol, $91.0 \%$ of the interaction forces between furfural and the solvent will be due to the formation of hydrogen bonds with misfit forces contributing $6.8 \%$ to the excess enthalpy. Likewise, according to COSMORS calculations, the interaction with hydrogen bonds will be the main one in the dissolution of furfural by thymol, with a contribution of $82.0 \%$, while the contribution of misfit forces to the excess enthalpy was $17.6 \%$. Therefore, the dissolution of furfural in both terpenoids involves strong hydrogen bonds between the solute and the solvent, the occurrence of misfit forces being secondary, and van der Waals forces being practically negligible.

Contrarily, the solvents that are non-oxygenated alkanes or non-aromatic solvents, such as limonene, menthol, or citral, show higher activity coefficients due to the absence of acceptor hydrogen bond groups or $\pi$ electrons to solubilize the furfural. In addition, the eutectic solvents formed by fatty acid and thymol suggest an increase in the extraction yield of furfural with a shorter alkyl chain, due to the increment of the nonpolarity with the alkyl chain length. 


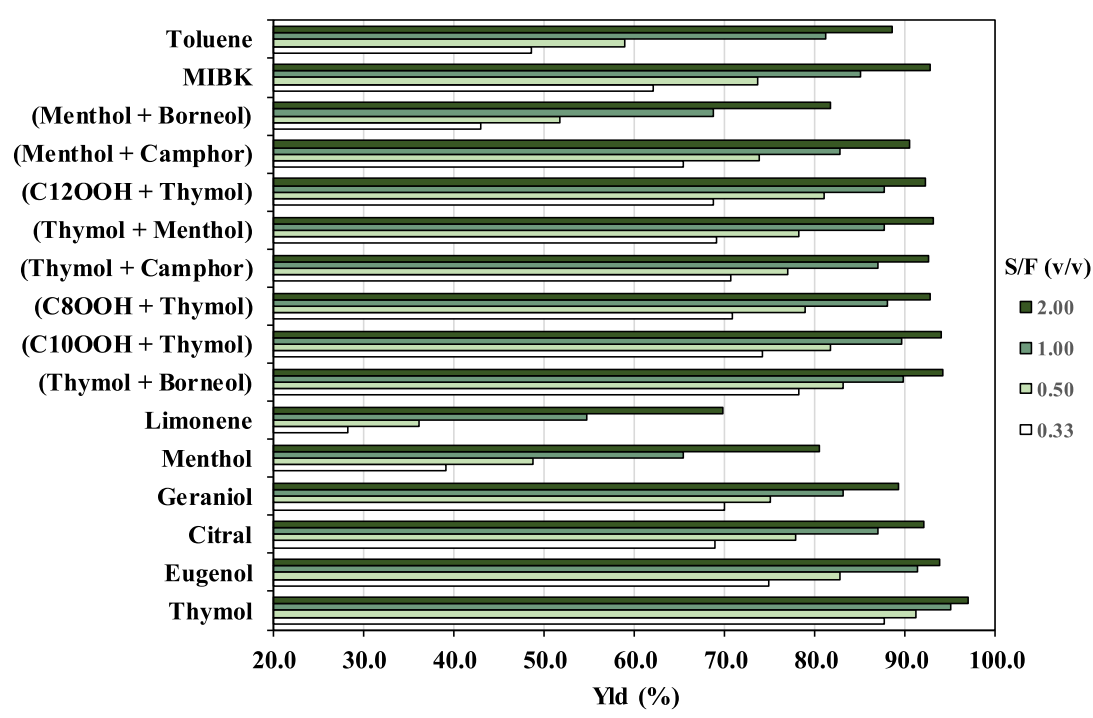

Figure 2. Furfural extraction yields obtained with the conventional solvents (toluene and MIBK), eutectic solvents (menthol + borneol, menthol + camphor, $\mathrm{C}_{12} \mathrm{OOH}+$ thymol, thymol + menthol, thymol + camphor, $\mathrm{C}_{8} \mathrm{OOH}+$ thymol, $\mathrm{C}_{10} \mathrm{OOH}+$ thymol, and thymol + borneol), a terpene (limonene), and terpenoids (thymol, menthol, eugenol, geraniol, and citral) as a function of $S / F$ volume ratio at $323.2 \mathrm{~K}$.

To validate the COSMO-RS predictions, the solvents with purple bars in Figure 1 were selected for further experimentation to cover a wide range of activity coefficients at infinite dilution. The solvents considered were one terpene (limonene), five terpenoids (menthol, geraniol, citral, eugenol, and thymol), eight eutectic solvents $[($ menthol + borneol $)$, (menthol + camphor), $\left(\mathrm{C}_{12} \mathrm{OOH}+\right.$ thymol), (thymol + menthol), (thymol + camphor), $\left(\mathrm{C}_{8} \mathrm{OOH}+\right.$ thymol $)$, $\left(\mathrm{C}_{10} \mathrm{OOH}+\right.$ thymol $)$, and (thymol + borneol $\left.)\right]$, and the conventional solvents with the lowest activity coefficients of furfural at infinite dilution, MIBK and toluene.

Extraction of Furfural Using Terpenoids and Eutectic Solvents. As indicated previously, the extraction of furfural from aqueous solutions was carried out in vials at $323.2 \mathrm{~K}$. The yield of extraction (Yld) was calculated for each solvent using the following equation

$$
\text { Yld }(\%)=\frac{C_{\mathrm{F}, \mathrm{o}}^{\mathrm{aq}}-C_{F}^{\mathrm{aq}}}{C_{\mathrm{F}, \mathrm{o}}^{\mathrm{aq}}} \times 100
$$

where $C_{\mathrm{F}, \mathrm{o}}^{\mathrm{aq}}$ is the initial concentration of furfural in the feed solution and $C_{\mathrm{F}}^{\mathrm{aq}}$ refers to the concentration of furfural in the aqueous phase after the extraction. The extraction yields are presented in Figure 2 as a function of the solvent-to-feed volume ratio $(S / F)$.

Thymol exhibited the best extraction performance, followed by eugenol and the eutectic solvent (thymol + borneol). The furfural extraction yields achieved with thymol or eugenol were significantly higher than those obtained with MIBK, the best conventional solvent tested. This difference was more remarkable operating at $\mathrm{S} / \mathrm{F}=0.33$, conditions under which thymol and eugenol reached extraction yields of 88 and $75 \%$, respectively, in comparison with $62 \%$ for MIBK. The eutectic solvents showed high extraction yields, except in the case of (menthol + borneol), which showed a poor performance. This finding was in agreement with the low extraction yield found for menthol, which just surpassed the performance of limonene. In general, the COSMO-RS predictions were in agreement with the experimental data. Thymol and eugenol showed the highest potential for furfural extraction, improving the results obtained with MIBK. Of the eutectic solvents tested, (thymol + borneol) and (thymol $\left.+\mathrm{C}_{10} \mathrm{OOH}\right)$ showed the best yields of extraction, increasing the yields obtained with MIBK at each S/F. Nevertheless, these yields of extraction are lower than those obtained with thymol and eugenol. On the other hand, limonene and menthol were identified as the terpene and terpenoid with the lowest extraction yields, respectively.

Interestingly, the extraction yields of the best solvents did not increase significantly with the $S / F$ ratio: for example, the extraction yields of thymol and eugenol at $S / F=1.00$ were comparable or higher than the ones obtained at $S / F=2.00$ using any other solvent. On the other hand, limonene, menthol, and toluene exhibited poor behavior, but their extraction yields increased substantially when the $S / F$ ratio increased (particularly, when the $S / F$ ratio shifted from 0.50 to $1.00)$. Considering the high yields achieved with eugenol and thymol at an $S / F$ ratio of 1.00 , this $S / F$ ratio has been selected as the optimum ratio to minimize the solvent and equipment costs, ensuring high furfural extraction yields. Because of this, the rest of the experiments were performed at $S / F=1.00$.

Also, the fraction of solvent dissolved in the aqueous phase and the amount of water dissolved in the organic phase has been quantified for the two best terpenoids, eugenol and thymol, and the two conventional organic solvents, MIBK and toluene. In Table S3 of the Supporting Information, the mutual solubilities of water and the four solvents obtained for an $S / F$ ratio of 1.00 at $323.2 \mathrm{~K}$ are shown.

As can be seen, the solvent fraction in the aqueous phase is an order of magnitude lower for the two terpenoids (eugenol and thymol) than the value obtained for the commercial reference solvent, MIBK. Therefore, the solvent losses in the aqueous phase using both terpenes would be lower than those produced using MIBK. In the case of water solubility in the organic phase, the terpenes have also shown slightly lower values than those obtained for MIBK. On the contrary, toluene due to its less polar character has shown the lowest values of solubility of the solvent in the aqueous phase and of water in the organic phase. However, as previously described in Figure 2 , the less polar character of toluene also implies significantly 
lower furfural extraction yields than those obtained for the two best terpenoids and MIBK.

To assess the agreement between the COSMO-RS predictions and the experimental data, Figure 3 shows the correlation between the experimental extraction yields and the furfural extraction activity coefficients at infinite furfural dilution estimated with COSMO-RS.

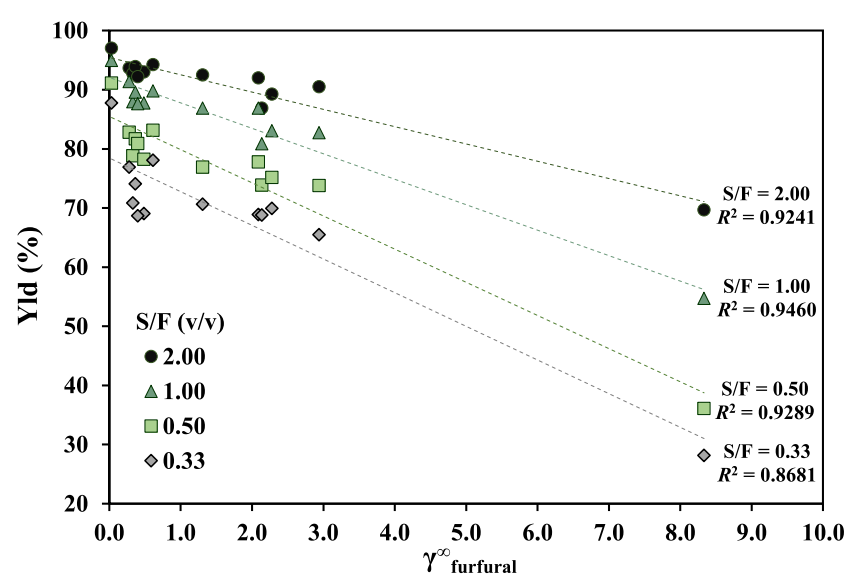

Figure 3. Correlation between the experimental extraction yields and the activity coefficients at infinite furfural dilution obtained using COSMO-RS.

In general, the COSMO-RS predictions were in good agreement with the experimental data, with less accurate predictions for the results obtained at the lower the $S / F$ ratio assayed. The solvents that showed low values of activity coefficient of furfural at infinite dilution in the predictions with COSMO-RS also achieved high experimental values of extraction yields of furfural. These results confirm that the COSMO-RS method is a suitable tool to predict the suitability of the considered solvents as agents for furfural production in biphasic reactors.

Extraction of Furfural under Reaction Conditions. Experiments of furfural extraction with thymol and eugenol, which are the best natural solvents tested, were performed to assess their behavior in a biphasic reactor at $443.2 \pm 0.5 \mathrm{~K}$. For comparison, similar experiments were carried out using the conventional solvents MIBK and toluene. Table 1 lists the extraction yields obtained operating at $S / F=1.00$.

Table 1. Furfural Extraction Yields Obtained in Experiments Performed in Vials (at 323.2 K) and in the Reactor at the Furfural Production Temperature (443.2 K) in the Absence or Presence of a Catalyst Acid $\left(0.1 \mathrm{M} \mathrm{H}_{2} \mathrm{SO}_{4}\right)$

\begin{tabular}{lccc} 
solvent & $\begin{array}{c}\text { vial at } 323.2 \mathrm{~K} \\
\text { Yld }(\%)\end{array}$ & $\begin{array}{c}\text { reactor at } \\
\text { 443.2 K Yld }(\%)\end{array}$ & $\begin{array}{c}\text { reactor at } 443.2 \mathrm{~K}+\text { acid } \\
\text { medium Yld }(\%)\end{array}$ \\
eugenol & 91.4 & 92.6 & 92.4 \\
thymol & 95.0 & 95.9 & 95.0 \\
toluene & 81.2 & 80.0 & 79.0 \\
MIBK & 85.1 & 86.8 & 87.0 \\
\hline
\end{tabular}

The data confirmed that the yields achieved under reaction conditions were close to the ones obtained in $8 \mathrm{~mL}$ vials, indicating that the screening of solvents operating in vials at 323.2 K was a strategy suitable for predicting their performance under reaction conditions $(443.2 \mathrm{~K}$ in the presence of $0.1 \mathrm{M}$ $\left.\mathrm{H}_{2} \mathrm{SO}_{4}\right)$. The extraction yields obtained at $443.2 \mathrm{~K}$ were slightly favored by the increase of the furfural solubility with temperature $^{25}$ and were scarcely affected by the presence of 0.1 $M$ sulfuric acid in reaction media, suggesting that the in situ extraction of furfural with the considered solvents is a suitable method for improving the results.

As mentioned in the Experimental Section, the chemical stability of thymol and eugenol after extraction at 323.2 and 443.2 K was analyzed by FTIR. The infrared spectra of both solvents are depicted in Figures S4 and S5. As the figure shows, the results suggest that the bands in both spectra do not display meaningful changes with the solvents used at $443.2 \mathrm{~K}$. Nonetheless, the $\mathrm{O}-\mathrm{H}$ band from 3600 to $3300 \mathrm{~cm}^{-1}$ due to the presence of water is slightly lower with both solvents used at $443.2 \mathrm{~K}$ than at $323.2 \mathrm{~K}$, due to the increase of the temperature and the decrease in water solubility.

To complete the study of the stability of terpenoids under reaction conditions, thymol and eugenol were analyzed before and after the extraction process by ${ }^{1} \mathrm{H}$ NMR. The spectra obtained are shown in Figures S6 and S7 of the Supporting Information. As can be seen, the signals observed before and after the extraction process are coincident, with only a slight increase in the signal corresponding to water $(2.8-3.1 \mathrm{ppm})$ due to the dissolution of a small amount of aqueous phase in the terpenoid together with the extracted furfural. Therefore, no changes in the chemical structure of the solvents were observed in either FTIR or ${ }^{1} \mathrm{H}$ NMR analyses. The use of terpenoids has an advantage over the use of eutectic solvents in a biphasic reactor. In the case of eutectic solvents, one of the components could be partially dissolved in water, changing the solvent composition, while in the case of terpenoids the composition of the solvent remains constant since it is a pure compound and no changes were observed in the signals corresponding to the terpenoids, eugenol and thymol.

The thermal stability of the solvents before and after furfural extraction was studied by TGA. The dynamic TGA curves obtained in the analysis of the fresh solvents and after use in the reactor are shown in Figures S8 and S9 of the Supporting Information. As can be seen, the only difference shown in the thermal stabilities is a slight weight loss at temperatures between 303.2 and $423.2 \mathrm{~K}$ in the case of the solvents used, due to the extraction of furfural and water in the organic phase. The decrease due to solvent evaporation (above $473.2 \mathrm{~K}$ ) is practically coincident in the fresh solvents and in those used in the extraction, thus confirming that solvent stability is not affected in the furfural extraction process under reaction conditions.

In conclusion, both solvents are chemically and thermally stable to carry out the extraction of furfural in a biphasic reactor at $443.2 \mathrm{~K}$, guaranteeing higher yields of extraction than the conventional solvents.

In Situ Production and Extraction of Furfural by Acidic Treatments of Xylose Solution in Biphasic Systems. Furfural was produced in biphasic media containing an acidic aqueous xylose solution and thymol or eugenol as the organic phase, operating at $S / F=1.00$ and $443.2 \mathrm{~K}$. For comparative purposes, similar experiments were performed using MIBK. Tables S4 and S5 of the Supporting Information show the composition of the aqueous and organic phases resulting from treatments performed in reactors with conventional or microwave heating, respectively.

Conversion of xylose for both conventional $(\mathrm{CH})$ and microwave heating (MW) is shown in Figure 4. A comparison between both biphasic systems using thymol or eugenol as the 


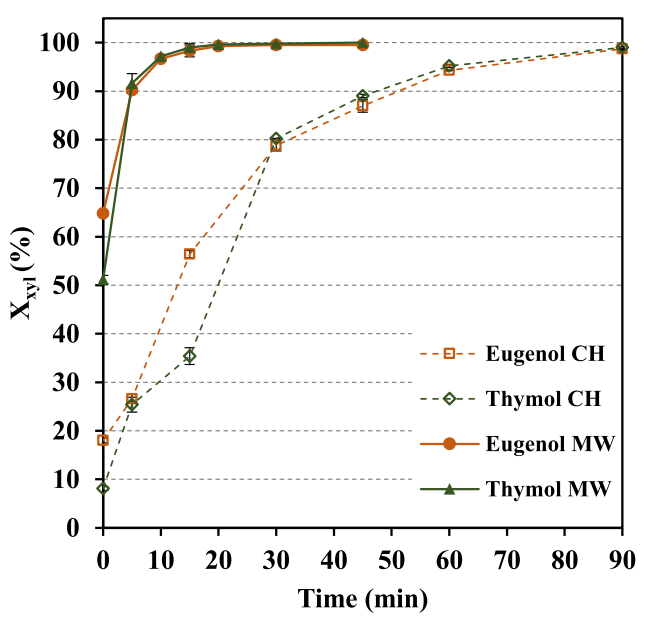

Figure 4. Conversion of xylose in biphasic media containing thymol or eugenol, operating in a reactor with $\mathrm{CH}$ or MW.

organic solvent showed similar conversions of xylose, thus demonstrating that this compound is not significantly affected by the presence of the organic phase. This behavior on the sugar reaction in aqueous media is in accordance with the reported literature. ${ }^{40}$ Xylose consumption in the aqueous phase occurs even in the heating stage and continues in the isothermal phase $(443.2 \mathrm{~K})$ as the reaction time increases. In the experiments performed by conventional heating, xylose reached conversions of nearly $90 \%$ between 45 and $60 \mathrm{~min}$ for both eugenol and thymol, respectively, while only $5 \mathrm{~min}$ are needed to reach that conversion by microwave heating. Thus, as expected, xylose conversion to furfural using microwave heating is significantly faster than using conventional heating.

Figure 5 shows the data determined for the furfural reaction yield, furfural selectivity, and xylose conversion in experiments performed in the microwave reactor. As shown in Figure 5a, the furfural reaction yield reached a maximum (72.8\%) after 10 min in media containing eugenol, in comparison with 65.8 and $51.8 \%$ when MIBK and thymol were employed as solvents, respectively. In assays with eugenol or thymol, prolonged reaction times resulted in decreased yields, which remained constant under the same conditions with MIBK as the solvent. This different behavior is ascribed to a possible coextraction of the acid by the natural solvents, as discussed later. Remarkably, high furfural reaction yields were achieved in media containing eugenol at reaction times of about $10 \mathrm{~min}$.

Regarding Figure 5b, xylose conversions and selectivity toward furfural using the three solvents selected for this step are shown together. As can be seen, the selectivity decreases more significantly after $10 \mathrm{~min}$ of experimentation, especially in the case of eugenol. One possible explanation could be the coextraction of the acid from the aqueous to the organic phase. This behavior is in accordance with Dietz et al., ${ }^{40}$ who reported a possible co-extraction of the acid catalyst in biphasic experiments related to furfural degradation when thymol was used as the extracting solvent. Furthermore, this furfural degradation was even higher when isophorone or cyclopentyl methyl ether was used. ${ }^{40}$

To reach a balance between xylose conversion, furfural reaction yield, and furfural selectivity, $10 \mathrm{~min}$ would be the optimal reaction time. The xylose conversion of $96.7 \%$, selectivity toward furfural of $75.3 \%$, and furfural reaction yield of $72.8 \%$ could be reached using eugenol. At this time, similar xylose conversions could be obtained using MIBK and thymol, but the values of furfural yield and selectivity would be substantially lower. Therefore, in the production of furfural from xylose in a biphasic reactor, the best performance was shown by eugenol with respect to the two solvents previously reported in the literature, MIBK and thymol. ${ }^{18,40}$

Furfural extraction yields calculated for each reaction time of experiments performed by $\mathrm{CH}$ and $\mathrm{MW}$ experiments (Tables S4 and S5) demonstrated that the extraction yield of furfural using thymol is between 95 and 97\%, while in the case of eugenol this percentage is slightly lower, between 93 and 94\%. These percentages obtained in the in situ reaction agree and even increase those obtained in the extraction experiments at the temperature of the reaction as listed in Table 1 . With respect to MIBK used as the reference, furfural extraction was slightly lower, around $90 \%$. In accordance with this last value, the reported furfural recovery in assays performed with MIBK at an $S / F$ of 1.00 was between 89.5 and $91.0 \%{ }^{18}$ In addition, the extraction yields of furfural obtained remained constant throughout the reaction. Therefore, considering the furfural selectivity and the furfural extraction and reaction yields, eugenol would sustainably improve the conventional solvents yields to extract furfural from its reaction medium from xylose.
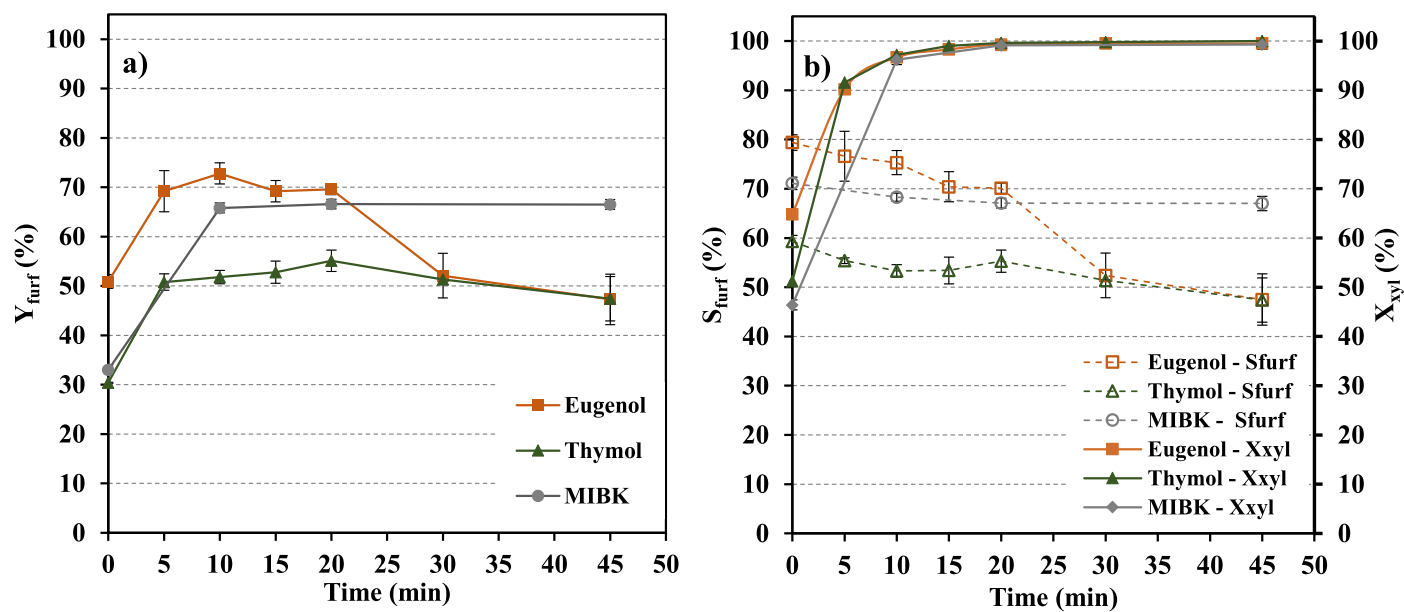

Figure 5. (a) Furfural reaction yield $\left(Y_{\text {furf }}\right)$. (b) Furfural selectivity $\left(S_{\text {furf }}\right)$ and conversion of xylose $\left(X_{\text {xyl }}\right)$ using thymol, eugenol, and MIBK (experiments performed in the microwave reactor). 
Simulation of Furfural Separation from Eugenol and MIBK by Distillation. As described in the Experimental Section, to study the technical feasibility of the proposed process for obtaining furfural using eugenol, a simulation of the separation process of furfural from eugenol and MIBK by distillation was carried out in Aspen Plus. The simulations were aimed at obtaining a furfural stream with a purity of $99.9 \%$ on a molar basis. Table 2 collects the energy consumption of both

Table 2. Results Obtained in the Simulation of the Separation by Distillation of Furfural Extracted by Eugenol and MIBK Using Aspen Plus

\begin{tabular}{lccc} 
solvent & reflux ratio & condenser duty $(\mathrm{kW})$ & reboiler duty $(\mathrm{kW})$ \\
eugenol & 1.13 & -49.8 & 929.1 \\
MIBK & 2.23 & -3053.8 & 1809.7 \\
\hline
\end{tabular}

systems, in the separation of $100 \mathrm{kmol} / \mathrm{s}$ of binary mixtures (furfural + eugenol) and (furfural + MIBK) with $2 \%$ of furfural on a molar basis.

In the case of eugenol, since its boiling point $(527.2 \mathrm{~K})$ is higher than that of furfural $(435.2 \mathrm{~K})$, furfural will be obtained in the distillate and the purified solvent will be obtained in the residue stream. On the contrary, using the reference solvent, MIBK, which has a boiling point of $389.2 \mathrm{~K}$, it is necessary to evaporate all the solvent to obtain it in the distillate. Therefore, the election of eugenol as the high-boiling-point solvent reduces the cooling and heating energy expenses to a large extent. Energy savings are $98.4 \%$ in cooling and $48.7 \%$ in heating because in the case of eugenol only $2 \%$ of the feed stream corresponding to furfural has to be obtained in the distillate. In addition to this, the regenerated eugenol leaves the distillation column above reaction temperature, imposing a clear advantage to minimize the heating expenses related to the reaction step. Thus, the use of eugenol as the solvent in a twophase reactor to obtain furfural could lead to an increase in the yields of furfural obtained with respect to the results using MIBK and also to a reduction in the costs associated with the subsequent process of separation of the solvent and the solute by distillation.

\section{CONCLUSIONS}

In this work, terpenes, terpenoids, and hydrophobic eutectic solvents have been assessed as agents for furfural recovery from aqueous solutions. A COSMO-RS prediction allowed the identification of the most favorable solvents for this purpose. Experimental extraction yields were correlated with the activity coefficients at infinite dilution predicted by the COSMO-RS method to get an insight into the performance of novel solvents with enhanced distribution ratios. This assessment allowed the identification of thymol, eugenol, and eutectic solvents containing thymol as promising candidates for furfural production, with comparative advantages over MIBK, considered as a reference solvent.

The solvents providing the highest extraction yields, thymol and eugenol, were evaluated for furfural extraction under reaction conditions typical for furfural. After confirming that temperature and acid hardly affected the extraction yield, additional experiments were performed to assess the in situ reaction and extraction of furfural from xylose. The best results were obtained operating with eugenol, which led to remarkable results in terms of furfural selectivity (75.3\%), furfural reaction yield $(72.8 \%)$, and extraction yield $(90.6 \%)$, improving the performance of MIBK. In addition, the separation of furfural from eugenol by distillation has been simulated, obtaining much lower energy costs than those required to separate furfural from the reference solvent, MIBK. The results achieved in this study confirm the potential of eugenol as a sustainable and efficient solvent for furfural production in biphasic reactors.

\section{ASSOCIATED CONTENT}

\section{Supporting Information}

The Supporting Information is available free of charge at https://pubs.acs.org/doi/10.1021/acssuschemeng.1c02798.

Description of chemicals; mass fractions of solvents in the aqueous phase and mass fraction of water in the organic phase in the extraction of furfural at $323.2 \mathrm{~K}$; results of in situ acidic treatment of pentoses in a biphasic system by conventional heating and microwave heating; sigma profiles of furfural and the solvents obtained from COSMOthermX; FTIR spectra of thymol and eugenol before and after heating; ${ }^{1} \mathrm{H}$ NMR spectra of eugenol and thymol before and after extraction of furfural under reaction conditions; and TGA of eugenol and thymol before and after extraction of furfural under reaction conditions (PDF)

\section{AUTHOR INFORMATION}

\section{Corresponding Author}

Marcos Larriba - Grupo de Catálisis y Procesos de Separación (CyPS), Departamento de Ingeniería Química y de Materiales, Universidad Complutense de Madrid, Madrid 28040, Spain; 잉oid.org/0000-0003-0058-6841; Phone: +34 91394 4135; Email: marcoslarriba@ucm.es

\section{Authors}

Andrés Cañada-Barcala - Grupo de Catálisis y Procesos de Separación (CyPS), Departamento de Ingeniería Química y de Materiales, Universidad Complutense de Madrid, Madrid 28040, Spain

Diego Rodríguez-Llorente - Grupo de Catálisis y Procesos de Separación (CyPS), Departamento de Ingeniería Química y de Materiales, Universidad Complutense de Madrid, Madrid 28040, Spain

Laura López - Departamento de Ingeniería Química, Universidade de Vigo, Facultade de Ciencias, Ourense 32004, Spain; CINBIO, Universidade de Vigo, Ourense 32004, Spain

Pablo Navarro - Departamento de Ingeniería Química, Universidad Autónoma de Madrid, Madrid 28049, Spain; (1) orcid.org/0000-0002-0017-3898

Elisa Hernández - Departamento de Ingeniería Química, Universidad Autónoma de Madrid, Madrid 28049, Spain

V. Ismael Águeda - Grupo de Catálisis y Procesos de Separación (CyPS), Departamento de Ingeniería Química y de Materiales, Universidad Complutense de Madrid, Madrid 28040, Spain

Silvia Álvarez-Torrellas - Grupo de Catálisis y Procesos de Separación (CyPS), Departamento de Ingeniería Química y de Materiales, Universidad Complutense de Madrid, Madrid 28040, Spain

Juan C. Parajó - Departamento de Ingeniería Química, Universidade de Vigo, Facultade de Ciencias, Ourense 32004, 
Spain; CINBIO, Universidade de Vigo, Ourense 32004, Spain; ๑ orcid.org/0000-0002-5610-6184

Sandra Rivas - Departamento de Ingeniería Química, Universidade de Vigo, Facultade de Ciencias, Ourense 32004, Spain; CINBIO, Universidade de Vigo, Ourense 32004, Spain

Complete contact information is available at: https://pubs.acs.org/10.1021/acssuschemeng.1c02798

\section{Notes}

The authors declare no competing financial interest.

\section{ACKNOWLEDGMENTS}

The authors are grateful to Comunidad Autónoma de Madrid for financial support of projects P2018/EMT-4341 and PR65/ 19-22441 and to Ministerio de Ciencia, Innovación y Universidades for financial support of the project CTM201784033-R. This work was supported by the Madrid Government (Comunidad de Madrid-Spain) under the Multiannual Agreement with Complutense University in the line Program to Stimulate Research for Young Doctors in the context of the V PRICIT (Regional Programme of Research and Technological Innovation). D.R.-L. and A.C.-B. thank Ministerio de Ciencia, Innovación y Universidades for awarding them FPU grants (FPU18/01536 and FPU19/01451). S.R. thanks Ministerio de Ciencia, Innovación y Universidades for her "Juan de la Cierva" contract (IJC2018-037665-I).

\section{REFERENCES}

(1) Yan, K.; Wu, G.; Lafleur, T.; Jarvis, C. Production, Properties and Catalytic Hydrogenation of Furfural to Fuel Additives and ValueAdded Chemicals. Renew. Sustain. Energy Rev. 2014, 38, 663-676.

(2) Cai, C. M.; Zhang, T.; Kumar, R.; Wyman, C. E. Integrated Furfural Production as a Renewable Fuel and Chemical Platform from Lignocellulosic Biomass. J. Chem. Technol. Biotechnol. 2014, 89, 2-10.

(3) Peleteiro, S.; Rivas, S.; Alonso, J. L.; Santos, V.; Parajó, J. C. Furfural Production Using Ionic Liquids: A Review. Bioresour. Technol. 2016, 202, 181-191.

(4) Li, X.; Jia, P.; Wang, T. Furfural: A Promising Platform Compound for Sustainable Production of C4 and C5 Chemicals. ACS Catal. 2016, 6, 7621-7640.

(5) Deng, F.; Amarasekara, A. S. Catalytic Upgrading of Biomass Derived Furans. Ind. Crops Prod. 2021, 159, 113055.

(6) Mariscal, R.; Maireles-Torres, P.; Ojeda, M.; Sádaba, I.; López Granados, M. Furfural: A Renewable and Versatile Platform Molecule for the Synthesis of Chemicals and Fuels. Energy Environ. Sci. 2016, 9, 1144-1189.

(7) Kabbour, M.; Luque, R. Furfural as a Platform Chemical: From Production to Applications; Elsevier B.V., 2019.

(8) Corma, A.; Iborra, S.; Velty, A. Chemical Routes for the Transformation of Biomass into Chemicals. Chem. Rev. 2007, 107, 2411-2502.

(9) Werpy, T.; Petersen, G. Top Value Added Chemicals from Biomass: Volume I -- Results of Screening for Potential Candidates from Sugars and Synthesis Gas; Office of Scientific and Technical Information (OSTI), 2004, Vol. 69.

(10) Lange, J.-P.; van der Heide, E.; van Buijtenen, J.; Price, R. Furfural-A Promising Platform for Lignocellulosic Biofuels. ChemSusChem 2012, 5, 150-166.

(11) Chheda, J. N.; Román-Leshkov, Y.; Dumesic, J. A. Production of 5-Hydroxymethylfurfural and Furfural by Dehydration of BiomassDerived Mono- and Poly-Saccharides. Green Chem. 2007, 9, 342-350.

(12) Esteban, J.; Vorholt, A. J.; Leitner, W. An Overview of the Biphasic Dehydration of Sugars to 5-Hydroxymethylfurfural and Furfural: A Rational Selection of Solvents Using COSMO-RS and Selection Guides. Green Chem. 2020, 22, 2097-2128.
(13) Alonso, D. M.; Bond, J. Q.; Dumesic, J. A. Catalytic Conversion of Biomass to Biofuels. Green Chem. 2010, 12, 1493-1513.

(14) Li, Q.; Sun, K.; Shao, Y.; Zhang, S.; Yan, Z.; Zhang, L.; Liu, Q.; Wang, Y.; Hu, X. Coordination of Acidic Deep Eutectic SolventChromium Trichloride Catalytic System for Efficient Synthesis of Fructose to 5-Hydroxymethylfurfual. Ind. Eng. Chem. Res. 2020, 59, $17554-17563$.

(15) Ji, H.; Zhu, J. Y.; Gleisner, R. Integrated Production of Furfural and Levulinic Acid from Corncob in a One-Pot Batch Reaction Incorporating Distillation Using Step Temperature Profiling. RSC Adv. 2017, 7, 46208-46214.

(16) Mandalika, A.; Runge, T. Enabling Integrated Biorefineries through High-Yield Conversion of Fractionated Pentosans into Furfural. Green Chem. 2012, 14, 3175-3184.

(17) Weingarten, R.; Cho, J.; Conner, W. C., Jr.; Huber, G. W. Kinetics of Furfural Production by Dehydration of Xylose in a Biphasic Reactor with Microwave Heating. Green Chem. 2010, 12, $1423-1429$.

(18) Rivas, S.; González-Muñoz, M. J.; Santos, V.; Parajó, J. C. Production of Furans from Hemicellulosic Saccharides in Biphasic Reaction Systems. Holzforschung 2013, 67, 923-929.

(19) Rivas, S.; Vila, C.; Santos, V.; Parajó, J. C. Furfural Production from Birch Hemicelluloses by Two-Step Processing: A Potential Technology for Biorefineries. Holzforschung 2016, 70, 901-910.

(20) Rivas, S.; Vila, C.; Alonso, J. L.; Santos, V.; Parajó, J. C.; Leahy, J. J. Biorefinery Processes for the Valorization of Miscanthus Polysaccharides: From Constituent Sugars to Platform Chemicals. Ind. Crops Prod. 2019, 134, 309-317.

(21) Mittal, A.; Black, S. K.; Vinzant, T. B.; O’Brien, M.; Tucker, M. P.; Johnson, D. K. Production of Furfural from Process-Relevant Biomass-Derived Pentoses in a Biphasic Reaction System. ACS Sustain. Chem. Eng. 2017, 5, 5694-5701.

(22) Xu, S.; Pan, D.; Wu, Y.; Song, X.; Gao, L.; Li, W.; Das, L.; Xiao, G. Efficient Production of Furfural from Xylose and Wheat Straw by Bifunctional Chromium Phosphate Catalyst in Biphasic Systems. Fuel Process. Technol. 2018, 175, 90-96.

(23) Molina, M. J. C.; Granados, M. L.; Gervasini, A.; Carniti, P. Exploitment of Niobium Oxide Effective Acidity for Xylose Dehydration to Furfural. Catal. Today 2015, 254, 90-98.

(24) Morais, E. S.; Freire, M. G.; Freire, C. S. R.; Coutinho, J. A. P.; Silvestre, A. J. D. Enhanced Conversion of Xylan into Furfural Using Acidic Deep Eutectic Solvents with Dual Solvent and Catalyst Behavior. ChemSusChem 2020, 13, 784-790.

(25) Dietz, C. H. J. T. Solvent Development for Recovery of Furfural and Hydroxymethylfurfural from Aqueous Biorefinery Solutions. Phd Thesis, Technische Universiteit Eindhoven: Netherlands, 2019.

(26) Lee, C. B. T. L.; Wu, T. Y. A Review on Solvent Systems for Furfural Production from Lignocellulosic Biomass. Renew. Sustain. Energy Rev. 2021, 137, 110172.

(27) Ghatta, A. A.; Wilton-Ely, J. D. E. T.; Hallett, J. P. Strategies for the Separation of the Furanic Compounds HMF, DFF, FFCA, and FDCA from Ionic Liquids. ACS Sustain. Chem. Eng. 2019, 7, 1648316492.

(28) Van Osch, D. J. G. P.; Dietz, C. H. J. T.; Van Spronsen, J.; Kroon, M. C.; Gallucci, F.; Van Sint Annaland, M.; Tuinier, R. A Search for Natural Hydrophobic Deep Eutectic Solvents Based on Natural Components. ACS Sustain. Chem. Eng. 2019, 7, 2933-2942.

(29) Phelps, T. E.; Bhawawet, N.; Jurisson, S. S.; Baker, G. A. Efficient and Selective Extraction of $99 \mathrm{~m} \mathrm{TcO} \mathrm{4-from} \mathrm{Aqueous}$ Media Using Hydrophobic Deep Eutectic Solvents. ACS Sustain. Chem. Eng. 2018, 6, 13656-13661.

(30) Silvestre, A. J. D.; Gandini, A. Terpenes: Major Sources, Properties and Applications. Monomers, Polymers and Composites from Renewable Resources; Elsevier, 2008, pp 17-38.

(31) Martins, M. A. R.; Silva, L. P.; Ferreira, O.; Schröder, B.; Coutinho, J. A. P.; Pinho, S. P. Terpenes Solubility in Water and Their Environmental Distribution. J. Mol. Liq. 2017, 241, 996-1002. 
(32) Schwab, W.; Fuchs, C.; Huang, F.-C. Transformation of Terpenes into Fine Chemicals. Eur. J. Lipid Sci. Technol. 2013, 115, 3-8.

(33) Zwenger, S.; Basu, C. Plant Terpenoids: Applications and Future Potentials. Biotechnol. Mol. Biol. Rev. 2008, 3, 1-7.

(34) Dejoye Tanzi, C.; Abert Vian, M.; Ginies, C.; Elmaataoui, M.; Chemat, F. Terpenes as Green Solvents for Extraction of Oil from Microalgae. Molecules 2012, 17, 8196-8205.

(35) Kumar, S. P. J.; Prasad, S. R.; Banerjee, R.; Agarwal, D. K.; Kulkarni, K. S.; Ramesh, K. V. Green Solvents and Technologies for Oil Extraction from Oilseeds. Chem. Cent. J. 2017, 11, 11.

(36) Thomsett, M. R.; Storr, T. E.; Monaghan, O. R.; Stockman, R. A.; Howdle, S. M. Progress in the Synthesis of Sustainable Polymers from Terpenes and Terpenoids. Green Mater. 2016, 4, 115-134.

(37) Rodríguez-Llorente, D.; Bengoa, A.; Pascual-Muñoz, G.; Navarro, P.; Águeda, V. I.; Delgado, J. A.; Álvarez-Torrellas, S.; García, J.; Larriba, M. Sustainable Recovery of Volatile Fatty Acids from Aqueous Solutions Using Terpenoids and Eutectic Solvents. ACS Sustain. Chem. Eng. 2019, 7, 16786-16794.

(38) Rodríguez-Llorente, D.; Cañada-Barcala, A.; Muñoz, C.; Pascual-Muñoz, G.; Navarro, P.; Santiago, R.; Águeda, V. I.; Álvarez-Torrellas, S.; García, J.; Larriba, M. Separation of Phenols from Aqueous Streams Using Terpenoids and Hydrophobic Eutectic Solvents. Sep. Purif. Technol. 2020, 251, 117379.

(39) Li, H.; Tamura, K. Ternary Liquid-Liquid Equilibria for (Water+terpene+1-Propanol or 1-Butanol) Systems at the Temperature 298.15K. Fluid Phase Equilib. 2008, 263, 223-230.

(40) Dietz, C. H. J. T.; Verra, M.; Verberkt, S.; Gallucci, F.; Kroon, M. C.; Neira D'Angelo, M. F.; Papaioannou, M.; Van Sint Annaland, M. Sequential and in Situ Extraction of Furfural from Reaction Mixture and Effect of Extracting Agents on Furfural Degradation. Ind. Eng. Chem. Res. 2019, 58, 16116-16125.

(41) Zainal-Abidin, M. H.; Hayyan, M.; Hayyan, A.; Jayakumar, N. S. New Horizons in the Extraction of Bioactive Compounds Using Deep Eutectic Solvents: A Review. Anal. Chim. Acta 2017, 979, 1-23.

(42) Paiva, A.; Craveiro, R.; Aroso, I.; Martins, M.; Reis, R. L.; Duarte, A. R. C. Natural Deep Eutectic Solvents-Solvents for the 21st Century. ACS Sustain. Chem. Eng. 2014, 2, 1063-1071.

(43) Verevkin, S. P.; Sazonova, A. Y.; Frolkova, A. K.; Zaitsau, D. H.; Prikhodko, I. V.; Held, C. Separation Performance of BioRenewable Deep Eutectic Solvents. Ind. Eng. Chem. Res. 2015, 54, 3498-3504.

(44) Liu, Y.; Friesen, J. B.; McAlpine, J. B.; Lankin, D. C.; Chen, S.N.; Pauli, G. F. Natural Deep Eutectic Solvents: Properties, Applications, and Perspectives. J. Nat. Prod. 2018, 81, 679-690.

(45) Dai, Y.; van Spronsen, J.; Witkamp, G.-J.; Verpoorte, R.; Choi, Y. H. Natural Deep Eutectic Solvents as New Potential Media for Green Technology. Anal. Chim. Acta 2013, 766, 61-68.

(46) Florindo, C.; Romero, L.; Rintoul, I.; Branco, L. C.; Marrucho, I. M. From Phase Change Materials to Green Solvents: Hydrophobic Low Viscous Fatty Acid-Based Deep Eutectic Solvents. ACS Sustain. Chem. Eng. 2018, 6, 3888-3895.

(47) Martins, M. A. R.; Crespo, E. A.; Pontes, P. V. A.; Silva, L. P.; Bülow, M.; Maximo, G. J.; Batista, E. A. C.; Held, C.; Pinho, S. P.; Coutinho, J. A. P. Tunable Hydrophobic Eutectic Solvents Based on Terpenes and Monocarboxylic Acids. ACS Sustain. Chem. Eng. 2018, 6, 8836-8846.

(48) Abranches, D. O.; Martins, M. A. R.; Silva, L. P.; Schaeffer, N.; Pinho, S. P.; Coutinho, J. A. P. Phenolic Hydrogen Bond Donors in the Formation of Non-Ionic Deep Eutectic Solvents: The Quest for Type V DES. Chem. Commun. 2019, 55, 10253-10256.

(49) Eckert, F.; Klamt, A. Fast Solvent Screening via Quantum Chemistry: COSMO-RS Approach. AIChE J. 2002, 48, 369-385.

(50) Klamt, A.; Jonas, V.; Bürger, T.; Lohrenz, J. C. W. Refinement and Parametrization of COSMO-RS. J. Phys. Chem. A 1998, 102, 5074-5085.

(51) Godbout, N.; Salahub, D. R.; Andzelm, J.; Wimmer, E. Optimization of Gaussian-Type Basis Sets for Local Spin Density Functional Calculations. Part I. Boron through Neon, Optimization Technique and Validation. Can. J. Chem. 1992, 70, 560-571.
(52) Klamt, A.; Reinisch, J.; Eckert, F.; Hellweg, A.; Diedenhofen, M. Polarization Charge Densities Provide a Predictive Quantification of Hydrogen Bond Energies. Phys. Chem. Chem. Phys. 2012, 14, 955963.

(53) Martins, M. A. R.; Silva, L. P.; Schaeffer, N.; Abranches, D. O.; Maximo, G. J.; Pinho, S. P.; Coutinho, J. A. P. Greener TerpeneTerpene Eutectic Mixtures as Hydrophobic Solvents. ACS Sustain. Chem. Eng. 2019, 7, 17414-17423.

(54) Lin, S.-T.; Sandler, S. I. A Priori Phase Equilibrium Prediction from a Segment Contribution Solvation Model. Ind. Eng. Chem. Res. 2002, 41, 899-913. 